\title{
Multi-channels Statistical and Morphological Features based Mitosis Detection in Breast Cancer Histopathology
}

\author{
Humayun Irshad $^{1}$, Ludovic Roux ${ }^{1}$, Daniel Racoceanu ${ }^{2}$
}

\begin{abstract}
Accurate counting of mitosis in breast cancer histopathology plays a critical role in the grading process. Manual counting of mitosis is tedious and subject to considerable inter- and intra-reader variations. This work aims at improving the accuracy of mitosis detection by selecting the color channels that better capture the statistical and morphological features having mitosis discrimination from other objects. The proposed framework includes comprehensive analysis of first and second order statistical features together with morphological features in selected color channels and a study on balancing the skewed dataset using SMOTE method for increasing the predictive accuracy of mitosis classification. The proposed framework has been evaluated on MITOS data set during an ICPR 2012 contest and ranked second from 17 finalists. The proposed framework achieved $74 \%$ detection rate, $70 \%$ precision and $72 \%$ F-Measure. In future work, we plan to apply our mitosis detection tool to images produced by different types of slide scanners, including multi-spectral and multi-focal microscopy.
\end{abstract}

\section{INTRODUCTION}

According to Nottingham Grading System [1], the well know international grading system for breast cancer recommended by the World Health Organization, mitotic count is one of the main factors in breast cancer grading. Indeed, mitotic count provides clues to estimate the proliferation and the aggressiveness of the tumor, representing a critical step in histological grading of several types of cancer. In clinical practice, the pathologists count mitosis after a tedious microscopic examination of hematoxylin and eosin (H\&E) stained tissue slides at high magnification, usually $40 \mathrm{X}$. The area visible in the microscope under a $40 \mathrm{X}$ magnification lens is called a high power field (HPF). This mitotic counting process is cumbersome and often subject to sampling bias due to massive histological images. This results in considerable inter- and intra-reader variation of up to $20 \%$ between central and institutional reviewers in tumor prognosis [2].

In histopathological image analysis, mitosis detection is a difficult task having to cope with several challenges such as irregular shaped objects, artifacts and unwanted objects, inherent to slide preparation and acquisition. Mitosis has four main evolution phases, each phase having different shape and texture. It is also observed that artifacts produce objects, which look similar to mitosis. As a result, there is no simple way to detect mitosis based on shape and pixels values. In addition, the major problem is the very low density of mitosis in a single HPF.

*This work is supported in part by the French National Research Agency ANR, Project MICO under reference ANR-10-TECS-015.

${ }^{1} \mathrm{H}$. Irshad and L. Roux are with the University Joseph Fourier, Grenoble 1, France. IPAL UMI CNRS

${ }^{2} \mathrm{D}$. Racoceanu is with the University Pierre and Marie Curie, Paris, France. IPAL UMI CNRS
The framework proposed in this study, aims at improving the accuracy of mitosis detection, by selecting the color channels that better capture the statistical and morphological features allowing robust mitosis discrimination from other similar objects (nuclei).

The reminder of the paper is organized as follows. Section II presents an overview for detection methods related to this research work. Section III describes the proposed framework for mitosis detection. Experimental results to demonstrate the effectiveness of our mitosis detection framework with different classifiers are presented in section IV. Finally, the concluding remarks with future work are given in section $\mathrm{V}$.

\section{PREVIOUS WORKS}

In literature of histopathology, there are very few research studies specifically dedicated to automated mitosis detection. Sertel et al. [3] developed a computer-aided system based on pixel-level likelihood functions and 2-step componentbased thresholding for mitotic counting in digitized images of neuroblastoma tissue and resulted in $81 \%$ of detection rate and $12 \%$ false positive rate. Anari et al. [4] proposed fuzzy cmean clustering algorithm along with ultra-erosion operation in Lab color space for detection of proliferative nuclei and mitosis index in IHC images of meningioma. Roullier et al. [5] proposed a multi-resolution unsupervised clustering driven by domain specific knowledge that resulted in more than $70 \%$ sensitivity and $80 \%$ specificity. The extraction of textural features from nuclear regions represents the variation in chromatin arrangement and, hence different stages in the nuclei cycle [6]. The diagnostic importance of nuclei texture has been widely studied [7]; yet recent work exploring nuclei classification of meningioma subtype in H\&E images via analysis of nuclear texture has been limited to one color channel [8].

The proposed framework is an extension of texture framework [9] for mitosis detection in RGB color space. We address some of the major shortcomings of previous works, including (1) comprehensive analysis of statistical features like Haralick features, run length features in different color spaces rather than single color space [9], (2) combining statistical features with morphological features in order to discriminate mitosis from other nuclei, and (3) exploring over-sampling method for balancing the skewed dataset by increasing the number of minority class to improve the predictive accuracy of mitosis classification. The improved framework include: (1) a robust multi-channel statistical features extraction which integrates segmented nuclei features at 
a variety of color spaces, (2) nuclei features describing both nuclear morphology, pixel information and texture that are able to quantify difference in mitosis and non-mitosis, and (3) studies of over-sampling methods to increase minority (mitosis) class size by interpolating between several minority class examples that lie together, which improve classification accuracy.

\section{PROPOSED FRAMEWORK}

We propose multi-channels statistical and morphological features combination strategy for automatic mitosis detection in $\mathrm{H} \& \mathrm{E}$ images. The proposed framework consists of three components: color channels selection, candidate detection and segmentation, and feature computation and classification, as shown in Fig. 1.

\section{A. Color Channels Selection}

The color representation plays an important role in histological image analysis since it carries usually more information than other features of a given color image [10]. For instance, different color space transformations have been applied to increase the separability between nuclei and nonnuclei during nuclei detection, segmentation and classification. In addition, different color models are proposed to separate a color into more useful components that bring new information to the system. In the proposed framework, our goal is to investigate the various color channels of different color models and select those channels having better pixels and texture information for mitosis detection. We convert RGB images into three other color models like HSV (more intuitive for human perception), Lab and Luv(uniform color separation). These four color models present common color models studied in histopathology. By doing histogram analysis of mitosis region with background regions in all channels of RGB, HSV, Lab and Luv color models, the selected channels are red, blue, V(HSV), L(Lab) and L(Luv).

\section{B. Candidate Detection and Segmentation}

In $\mathrm{H} \& \mathrm{E}$ stained images, nuclear region appears as hue of blue while extracellular material have hues of pink. In order to reduce the complexity by integrating Laplacian of Gaussian (LoG) responses, the RGB images are transformed into new image called blue-ratio (BR) image to accentuate the nuclear region [11].

$$
B R=\frac{100 * B}{1+R+G} * \frac{256}{1+B+R+G}
$$

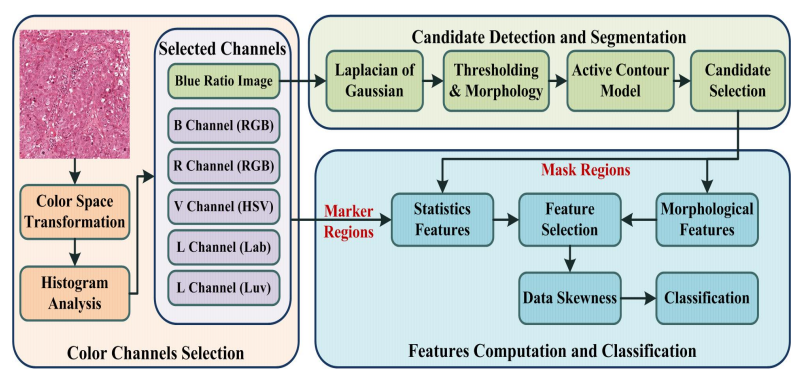

Fig. 1. The Proposed Framework where $\mathrm{R}, \mathrm{B}$ and $\mathrm{G}$ are blue, red and green channels of RGB color model, respectively. On BR image, we computed LoG responses that discriminate the nuclei region from the background region, hence assisting in detection of candidate for mitosis detection. Then, we perform binary thresholding followed by morphological processing to eliminate too small regions and fill holes. We observe that the thresholding with morphological operations based segmentation procedure described above, does not produce consistent boundaries. Later, we use the active contour models with level-set implementation for refining the nuclei boundary. Finally, we select the list of candidates by filtering on size of candidates.

\section{Feature Computation and Classification}

For each candidate, we extract two sets of quantitative morphological and statistical image features. The morphological features, computed on the segmented region of each candidate, are area, roundness, elongation, perimeter and equivalent spherical perimeter. These morphological features reflect the phenotypic information of mitotic nuclei. Using pixel intensity information of the selected color channels including BR image, we extract five first order statistical features including mean, median, variance, kurtosis and skewness of each segmented candidate. This results in 30 first order statistical features of each candidate.

Using the mask of each candidate to restrict texture analysis to relevant region, Haralick co-occurrence (HC) [12] and run-length (RL) [13] features are computed with one displacement vector in four directions $\left(0^{\circ}, 45^{\circ}, 90^{\circ}, 135^{\circ}\right)$ for all the selected channels. These texture features are rotationally invariant. So by making average in all four directions, the eight computed HC features are correlation, cluster shade, cluster prominence, energy, entropy, hara-correlation, inertia and difference moment and the ten computed RL features are SRE, LRE, LGRE, HGRE, SRLGE, SRHGE, LRLGE, LRHGE, GLNU and RLNU. This task is repeated for each selected channels resulting in 48 Haralick and 60 run-length features for each candidate.

Conceptually, a large number of descriptive features are highly desirable for classification of object as mitosis or non-mitosis. However, when use all computed features (i.e. 143 features) for classification of candidate as mitosis or non-mitosis, the classification performance is poor. Some features are irrelevant for classification and some features are redundant that represents duplication of features and does not provide additional class discriminatory information, degrading the classification performance. Later, we use consistency subset evaluation method [14] to select a subset of features that maximize the consistency in the class values. We evaluate the worth of subsets of features by the level of consistency in the class values using the projection of subset of features from training dataset. The consistencies of these subsets are not less than that of the full set of features. At last, we used these subsets in conjunction with a hill climbing search method, augmented with backtracking value 5, which looks for the smallest subset with consistency equal to that of the full set of features. This procedure 
achieved $86 \%$ reduction in the dimensionality of features set by selecting 20 features. The selected features contained two morphological features (area and equivalent spherical perimeter), eight first order statistical features (median in BR image, blue and L (Lab) channels, variance in BR image and blue channel, kurtosis in red and blue channels, and skewness in blue channel, five CM features; which are energy in BR image, difference moment in red and blue channels, cluster shade and hara-correlation in V (HSV) channel; and five RL features; which are HGRE in BR image, red channel and LGRE in red, blue and $\mathrm{L}(\mathrm{Lab})$ channel. The selected features set is used to train different classifier like random forest (RF) classifier, an ensemble classifier consisting of many decision trees, linear SVM (L-SVM) and non-linear SVM (NL-SVM) classifier [15]. Throughout the experiments, the parameters used in RF classifier are MaxDepth $=10$ and NumOfTree $=10$. We use L2-loss support vector machine for L-SVM classifier with bias $=1$, cost $=1$ and eps $=0.01$. For NLSVM classifier, we use rbf kernel with degree of kernel $=3$, eps $=0.001$ and loss $=0.1$.

\section{EXPERIMENT RESULTS}

We evaluated the proposed framework on MITOS database [16]. A total of five H\&E stained breast cancer biopsy cores from 5 patients were scanned using a high resolution whole slide Aperio scanners at 40X optical magnification in the Pitié-Salpêtrière hospital in Paris, France. The pathologists selected a total 50 HPFs from five whole slides, ten from each slide. These 50 HPFs contain a total of 326 mitosis. The training and testing set consisted of 35 and 15 HPFs, containing 226 and 100 mitosis, respectively. We compare the results of different classifiers with GT information provided along with the dataset. The metrics used to evaluate the mitosis detection include: number of true positives (TP), number of false positives (FP), true positive rate (TPR), positive predictive value (PPV) and F-Measure.

\section{A. Experiment 1: Classification of raw data}

For training classifiers, we used 35 HPFs containing 226 mitosis and 16290 non-mitosis. We found $100 \%$ accuracy on training dataset using RF classifier. On testing dataset, the candidate detection and segmentation phase detected 6238 candidates, containing $88 \mathrm{GT}$ mitosis from a total of $100 \mathrm{GT}$ mitosis. In candidate detection phase, we tried to get as many candidates as possible in order to prevent early phase mitosis missing. Therefore, among the entire candidate set, 6150 were non-mitosis in testing dataset. This phase generated a large number of non-mitosis and missed 12 GT mitosis.

Using all statistical features with RF classifier, we got a few number of TP with a large number of FP. By including morphological features with all statistical features, number of FP reduced with small increase in TP. Later, we applied different classifiers including RF, linear and non-linear SVM, on selected statistical and morphological features. As compared with linear SVM, the experiments with non-linear SVM resulted in better performances in terms of less FP but less TP as well. When we used selected features with RF classifier, we achieved higher TP with lower FP and resulted $72 \%$ TPR, 68\% PPV and 70\% F-Measure.

\section{B. Experiment 2: Classification of skewed data}

Later, we apply synthetic minority over-sampling technique (SMOTE) [17] on training set to increase the number of mitosis in order to reduce bias of classifiers towards non-mitosis class. This over-sampling approach creates extra synthetic training data for minority class by operating in feature space rather than data space. Depending upon the amount of over-sampling required, neighbors from the $\mathrm{k}$ nearest neighbors are randomly chosen. In our experiment, the amount of over-sampling needed is $200 \%$, only two neighbors from the five nearest neighbors are chosen and one sample is generated in the direction of each. In addition, down-sampling is applied on non-mitosis class which resulted 30\% reduction. The new training set includes 452 mitosis and 11400 non-mitosis. By using new training set, we improve the classification accuracy and reduce the number of FP as shown in Table I. The proposed framework got highest mitosis detection with 74\% TPR and overall ranked second in ICPR contest 2012 from a list of 17 finalists [16] and their results is shown in Fig. 2. The performance of the proposed framework is presented in the form of Receiver Operating Characteristic (ROC) Curve. The area under curve (AUC) on the training set is $100 \%$ and for testing set, the AUC is about 73.58\% (showing in Fig 3). Fig. 4 shows an example of mitosis detection framework result showing green circle are TP, blue circle is FP and yellow circle is FN using selective features with RF classifier.

\section{DISCUSSION}

The integration of statistical features with morphological features at various color channels improves mitosis detection with better detection rate than other methods during ICPR contest 2012 [16]. In experiment 1, we found that multichannels statistical features with nuclear morphology have better mitosis discrimination from other objects. In addition, we also found that the RF classifier has better results as compared to other classifiers, because of unbalancing error in class population datasets. One of the parameters that

TABLE I

Classification Result $(\mathrm{GT}=100)$.

\begin{tabular}{lccccc}
\hline Experiments & TP & FP & TPR & PPV & F-Measure \\
\hline $\begin{array}{l}\text { Statistical features } \\
\text { with RF }\end{array}$ & 50 & 26 & $50 \%$ & $66 \%$ & $57 \%$ \\
All features with RF & 52 & $\mathbf{2 0}$ & $52 \%$ & $\mathbf{7 2 \%}$ & $60 \%$ \\
\hline \multicolumn{7}{c}{ Experiment 1: Selective features without skewness of data } \\
L-SVM Classifier & 70 & 68 & $70 \%$ & $51 \%$ & $59 \%$ \\
NL-SVM Classifier & 61 & 35 & $61 \%$ & $64 \%$ & $62 \%$ \\
RF Classifier & 72 & 34 & $72 \%$ & $68 \%$ & $70 \%$ \\
\hline \multicolumn{5}{l}{ Experiment 2: Selective features with skewness of data } \\
L-SVM Classifier & 72 & 58 & $72 \%$ & $55 \%$ & $63 \%$ \\
NL-SVM Classifier & 65 & 32 & $65 \%$ & $67 \%$ & $65 \%$ \\
RF Classifier & $\mathbf{7 4}$ & 32 & $\mathbf{7 4 \%}$ & $70 \%$ & $\mathbf{7 2 \%}$ \\
\hline
\end{tabular}




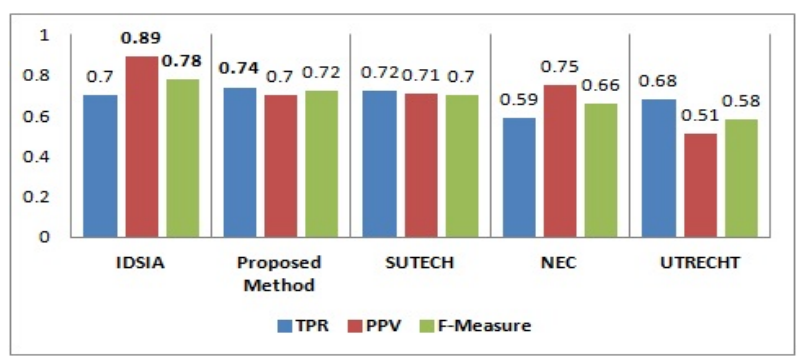

Fig. 2. ICPR 2012 Aperio Dataset Results [16]

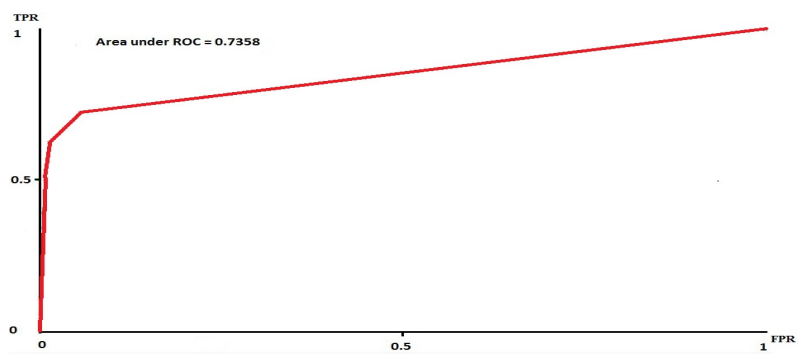

Fig. 3. The ROC Curve of the proposed framework on testing set.

mostly affect our experiments is the unbalanced training set having a huge number of non-mitosis compared to the small number of mitosis. Indeed, most of the classifiers are biased toward non-mitosis, which resulted high FP and low TP. In experiment 2, the use of SMOTE on training set in addition to down-sampling of non-mitosis class shows slightly better detection rate together with a lower rate of FP. The SMOTE method effectively forces the decision region of the minority class to become more general that eventually reduce the biasness of non-mitosis class and resulted in improve performance of classification. The missed mitosis normally have small in size and few nuclear material. These mitosis are also missed by all the methods during mitos contest 2012. The proposed framework is developed using insight toolkit (ITK) [18] and have average time complexity 30 minute per HPF on Intel(R) Core(TM) i5-2500 CPU @ $3.30 \mathrm{GHz}$ with $4 \mathrm{~GB}$ memory.

\section{CONCLUSION}

An automated mitosis detection framework for $H \& E$ images based on multi-channel statistical and morphological features has been proposed. Specifically, our multi-

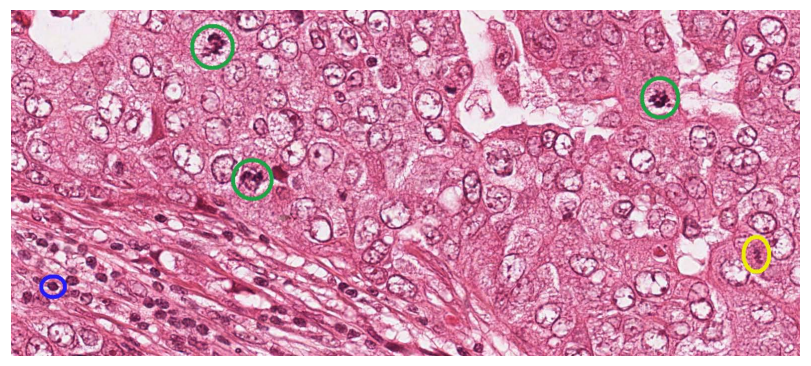

Fig. 4. Mitosis Detection framework result (green circles are TP, blue circle is FP and yellow circle is FN channel features extraction scheme uses segmented regions and extracts nuclei features in different color channels, a highly efficient model for capturing texture features for nuclei discrimination. The use of SMOTE on training dataset in addition to down-sampling of non-mitosis class shows slightly better mitosis detection rate together with a lower rate of FP. In future work, we plan to use color deconvolution as preprocessing and hough transform or local extrema based candidate detection in order to reduce the number of candidates. We also plan to apply our mitosis detection tool to images produced by different types of slide scanners, including multi-spectral and multi-focal microscopes.

\section{ACKNOWLEDGEMENT}

We acknowledge the Pathology Department of La PitiéSalpêtrière Hospital, leaded by Professor Frédérique Capron, for the knowledge provided and the rigorous data preparation.

\section{REFERENCES}

[1] C.W. Elston and I.O. Ellis, "Pathological prognostic factors in breast cancer. the value of histological grade in breast cancer experience from a large study with long-term follow-up," Histopathology, vol. 41, pp. $151,2002$.

[2] H.A. Teot, R. Sposto, A. Khayat, S. Qualman, G. Reaman, and D. Parham, "The problems and promise of central pathology review development of a standardized procedure for the children oncology group," Pediatr. Dev. Pathol., vol. 10, pp. 199-207, 2007.

[3] O. Sertel, U.V. Catalyurek, H. Shimada, and M.N. Gurcan, "Computeraided prognosis of neuroblastoma detection of mitosis and karyorrhexis cells in digitized histological images," in $E M B C, 2009$.

[4] V. Anari, P. Mahzouni, and R. Amirfattahi, "Computer-aided detection of proliferative cells and mitosis index in immunohistichemically images of meningioma," in MVIP, 2010.

[5] V. Roullier, O. Lezoray, V.T. Ta, and A. Elmoataz, "Mitosis extraction in breast-cancer histopathological whole slide images," in $A d v$. in Visual Computing. 2010.

[6] B. Weyn and et al., "Automated breast tumor diagnosis and grading based on wavelet chromatin texture description," Cytometry, vol. 33, pp. 32-40, 1997.

[7] W. Wang, J.A. Ozolek, and G.K. Rohde, "Detection and classification of thyroid follicular lesions based on nuclear structure from histopathology images," Cytometry, vol. 77A, pp. 485-94, 2010.

[8] O.S. Al-Kadi, "Texture measures combination for improved meningioma classification of histopathological images," Pat. Recog., vol. 43, pp. 2043-53, 2010.

[9] H. Irshad, S. Jalali, L. Roux, D. Racoceanu, J.H. Lim, G.L. Naour, and F. Capron, "Automated mitosis detection using texture, sift features and hmax biologically inspired approach," in HIMA@MICCAI, 2012.

[10] M. Mete and U. Topaloglu, "Statistical comparison of color modelclassifier pairs in hematoxylin and eosin stained histological images," in IEEE Symposium on CIBCB, 2009, pp. 284-91.

[11] H. Chang, L.A. Loss, and B. Parvin, "Nuclear segmentation in h\&e sections via multi-reference graph-cut (mrgc)," in ISBI, 2012.

[12] R.M. Haralick, K. Shanmugam, and I.H. Dinstein, "Textural features for image classification," IEEE Trnas. on Systems, Man and Cybernetics, vol. 3, pp. 610-21, 1973.

[13] M.M. Galloway, "Texture analysis using gray level run lengths," CGIP, vol. 4, pp. 172-9, 1975.

[14] H. Liu and R. Setiono, "A probabilistic approach to feature selection - a filter solution," in ICML, 1996, pp. 319-27.

[15] Weka, "Url http://www.cs.waikato.ac.nz/ml/weka," .

[16] MITOS contest 2012, "http://ipal.cnrs.fr/icpr2012," .

[17] N.V. Chawla, K.W. Bowyer, L.O. Hall, and W.P. Kegelmeyer, "Smote: Synthetic minority over-sampling technique," J. Art. Int. Res., vol. 16, pp. 321-57, 2002.

[18] Insight Toolkit (ITK), "http://www.itk.org/," . 